Jean-Antoine Caravolas, Histoire de la didactique des langues au siècle des Lumières. Précis et anthologie thématique, Montreal-Tübingen, Les Presses de l'Université de Montréal-Gunter Narr Verlag, 2000, $544 \mathrm{p}$.

Nadia Minerva

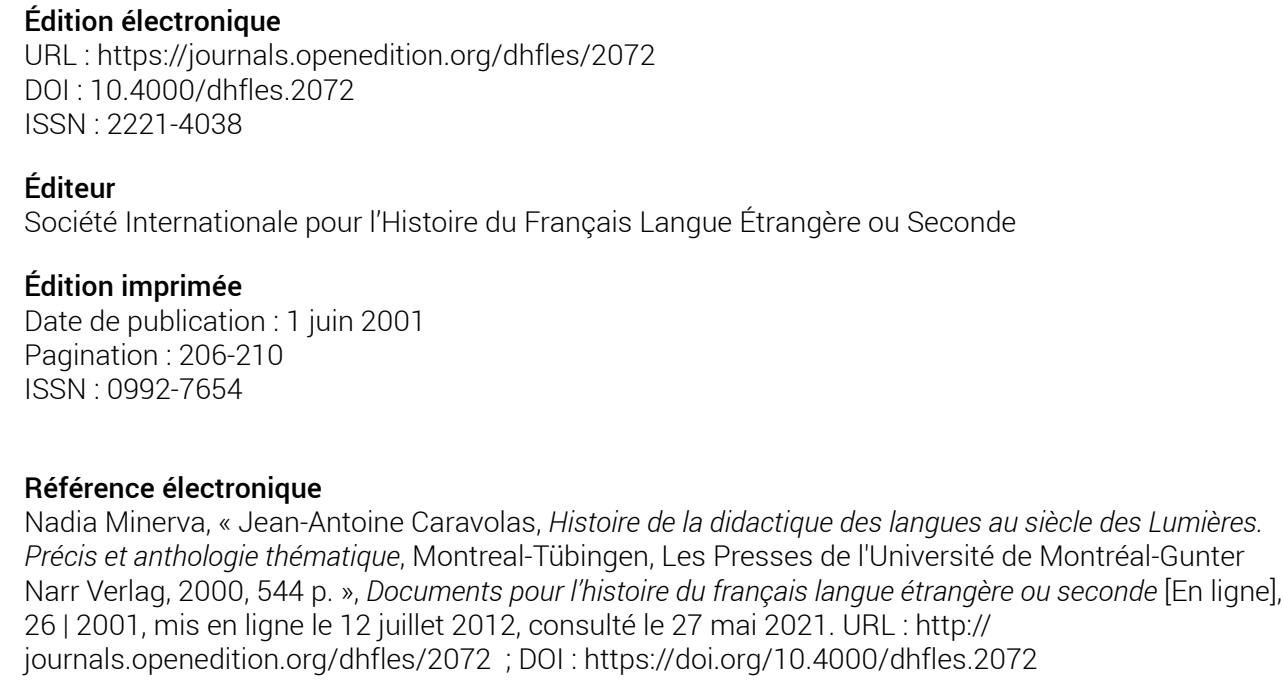

Ce document a été généré automatiquement le 27 mai 2021.

(c) SIHFLES 


\title{
Jean-Antoine Caravolas, Histoire de la didactique des langues au siècle des Lumières. Précis et anthologie thématique, Montreal-Tübingen, Les Presses de l'Université de Montréal- Gunter Narr Verlag, 2000, 544 p.
}

\author{
Nadia Minerva
}

1 Après le premier tome de la Didactique de 1994, Jean Caravolas poursuit sa réflexion sur l'histoire de l'enseignement des langues - classiques et modernes - en étendant son enquête au XVIII siècle : entreprise gigantesque pour laquelle il a eu recours à une documentation, patiemment recueillie, sur une cinquantaine d'auteurs de grammaire et de pédagogues exemplaires de toute la civilisation occidentale, en deçà et au-delà de l'Atlantique : de l'Angleterre à l'Italie, de la Péninsule Ibérique aux Balkans, de l'Autriche à la Scandinavie, du Canada aux États-Unis... L'intérêt en est inégal à cause des sources auxquelles l'auteur a pu puiser, aussi bien que de l'apport des pédagoguesgrammairiens de l'époque, parfois d'une originalité bien soulignée par Caravolas, parfois - plusieurs fois, pourrait-on dire - décevant, la scène étant occupée par les figurants aussi bien que par les protagonistes, ce qui restitue un tableau assez fidèle de la variété des acteurs en présence.

2 Quant au premier point, les études sur la didactique des langues abondent pour certains pays, telle l'Allemagne, grâce à des équipes que les Sihflésiens ont souvent eu l'occasion d'apprécier ; elles sont pauvres, voire inexistantes pour d'autres, dont la Russie, malgré l'ouverture promue par des souverains éclairés qui ont cultivé des relations suivies avec l'Occident européen : les liens étroits qui se sont tissés entre les cours européennes, entre les princes et les particuliers (dont la correspondance entre Catherine et les Philosophes n'est qu'un exemple) sont bien connus ; l'Europe des 
Lumières dans le siècle du cosmopolitisme et de l'universalité de la culture est l'espace d'une circulation d'idées et d'hommes dont les maîtres de langues ne sont qu'un aspect parmi tant d'autres. Cependant, si on connaît de mieux en mieux la réalité historique, politique ou littéraire de l'Est européen, la pédagogie des langues qui était pratiquée reste dans l'ombre, d'où l'intérêt du précis de Caravolas qui signale aux spécialistes un domaine presque vierge : comment apprenait-on les langues en Russie, en Pologne, dans les Balkans... ?

Quant à l'originalité de la pensée linguistique et pédagogique du XVIII siècle, l'Allemagne se signale par la variété et la richesse méthodologique dont ont fait preuve ses théoriciens et ses auteurs de manuels : entre approche philantropiniste et approche néo-humaniste, se dessine un vaste mouvement de réforme pédagogique qui investit l'enseignement des langues : méthode naturelle, méthode grammaticale et méthode mixte se partagent l'attention et constituent la matière d'un débat fécond, d'une effervescence d'idées, de méthodes, de stratégies didactiques qui, comme le relève Caravolas, placent l'Allemagne à la tête du mouvement européen pour le renouveau de la didactique des langues (p. 169).

4 À côté des noms illustres - pour leurs idées pédagogiques, pour leur contribution plus spécifique à la réflexion sur la didactique des langues, pour l'audience européenne de leurs théories linguistiques ou bien, enfin, pour la circulation supranationale de leurs manuels (Du Marsais, Pluche, Radonvilliers, Restaut, Rousseau, Diderot, Beauzée, Condillac, Lhomond, Faiguet, Batteux, Kant, Herder, Basedow, Des Pepliers, Cramer, Meidinger, Lévizac, Baretti, Perrin), bouge tout un monde de méconnus de l'enseignement linguistique tels - par exemple - les nombreux Italiens auteurs de manuels pour Anglais, Allemands, Français... ou les obscurs maitres anglais de français ou d'allemand... très actifs dans leur pays d'accueil, qui ont laissé des grammaires que Caravolas a su dénicher et offrir à l'attention des chercheurs : l'italien langue étrangère - pour ne fournir qu'un cas - n'a pas joui jusqu'à présent de l'intérêt qu'ont suscité d'autres domaines disciplinaires, notamment le FLE. Et pourtant son enseignement a été ininterrompu et souvent très qualifié, grâce à des maîtres d'exception (Baretti, entre autres).

5 Au fil des pages nous assistons à la naissance des exercices avec le Cours complet de langue françoise de Mauvillon (1754) ; nous sommes renseignés sur le " bureau typographique » de Dumas, inventé pour apprendre aux enfants à lire et à composer en latin et en français, sur les " fours grammaticaux », la " grammaire musicale » ou la " grammaire digitale " de Vallange qui exploite toutes les ressources possibles pour rendre l'apprentissages du latin moins rébarbatif aux petits Français (Art d'enseigner le latin aux petits enfants en les divertissant et sans qu'ils s'en aperçoivent, 1730) ; nous découvrons qu'au Canada, sous le régime français, les écoles pour filles étaient plus nombreuses que les écoles pour garçons ; nous faisons la connaissance de Noah Webster, « le précepteur des États-Unis », dont l'abécédaire (American Speller) est le plus grand best-seller pédagogique américain.

6 Le schéma des onze chapitres du "Précis » est rigoureusement respecté : aperçu historique, situation dans le domaine de l'éducation, enseignement des langues anciennes, enseignement des langues vivantes. Les régions géographiques analysées sont l'Angleterre, l'Allemagne, la France, la Belgique et les Pays-Bas, les États de l'Autriche, les Balkans, le Canada, les États-Unis ; deux regroupements pour les chapitres V et VIII surprennent : l'Europe du Sud (Italie, Espagne et Portugal) et 
l'Europe du Nord-Est (Pologne, Scandinavie et Russie). Quant au premier - pour ne prendre en compte que l'histoire du FLE sur laquelle nous sommes mieux renseignés -la vitalité et l'abondance des études sur les deux péninsules de l'Europe du Sud auraient pu peut-être autoriser un développement plus étendu (André Reboullet d'ailleurs le constatait déjà en 1995, dans le dossier qu'il a coordonné pour Documents $n^{\circ} 16$ : un déséquilibre frappant entre Nord et Sud avait atteint, depuis Brunot, les études sur la didactique du français à l'étranger; grâce à la SIHFLES ce déséquilibre s'était estompé et Reboullet n'avait pas manqué de montrer le renouveau des études récentes sur l'Italie et l'Espagne notamment). Le deuxième regroupement est justifié par Caravolas dans la conclusion du chapitre (p. 264) et dans la conclusion générale (p. 333) : la composition du Précis et la disparité de la longueur des chapitres ont été dictées par l'inégalité de l'évolution des idées linguistiques et pédagogiques dans les différents pays. La contribution de la Russie, de la Scandinavie et de la Pologne - précise-t-il - reste marginale et tributaire des idées et des méthodes allemandes et françaises.

7 Pour conclure mon rapide survol sur ce volume monumental, il me reste à signaler le chapitre IX, consacré à l'apprentissage des langues chez les Jésuites, l'anthologie thématique, le lexique des noms et la bibliographie qui, à la mesure de l'ouvrage, s'étend sur une quarantaine de pages. Quant à l'anthologie (pp. 343-476), les auteurs choisis par Caravolas abordent, à côté des sujets d'intérêt général (" Enseigner une langue étrangère ", "Qu'enseigner ? ", « Le rôle de la mémoire »...), des questions plus ponctuelles : l'apprentissage de la lecture, de l'écriture, de la prononciation... en témoignant d'une certaine pérennité de la problématique de l'enseignement des langues. Si les questions ne changent pas au fil des siècles, les réponses ne sont pas moins récurrentes - presque cycliques, pourrait-on enferrer - malgré la gamme très variée d'interprétations de l'acte d'enseigner et des mécanismes de l'apprentissage linguistique, et malgré la variété des propositions qui se sont suivies du XVIIIe siècle à nos jours : le grand dilemme reste en effet celui de toutes les époques. Enseignement/ apprentissage par les règles ou par l'usage ? Grammaire ou routine? L'ancien adage de Quintilien ne cesse de partager les linguistes et les didacticiens du XVIIIe siècle. En cela le siècle des Lumières n'a fait qu'amplifier un débat immortel. Pourrait-on en dresser un bilan?

En dépit de toutes les révolutions (philosophique, industrielle, américaine et française) qui agitent la société du XVIIIe siècle, aucun bouleversement significatif n'atteint la pédagogie des langues. Si la naissance de la langue maternelle comme discipline scolaire, l'évolution de la théorie pédagogique, la bifurcation des études secondaires en Allemagne, les débats sur la professionnalisation du métier d'enseignant, l'arrivée massive des femmes sur le marché du travail, les changements de la conception de la langue (toutes ces transformations et d'autres encore sont résumées par Caravolas dans sa conclusion) pouvaient faire présager une évolution « tumultueuse » de la didactique des langues, les innovations méthodologiques sont pourtant rares : enseignement formel de la langue maternelle; déclin de l'importance de la compétence oral en latin ; alternance pour les langues vivantes de la méthode grammaire-traduction et de la méthode de lecture. Si « à la fin du siècle des Lumières les différences entre la méthode d'enseignement des langues mortes et celle des langues vivantes disparaissent presque entièrement » (p. 337), les cours privés constituent encore le rempart d'un enseignement personnalisé, adapté aux besoins sociaux du milieu qui les sollicite, centré sur l'apprenant. Cette pratique que l'optique de la scolarisation privilégiée par 
Caravolas a quelque peu négligée, bien vivante et fructueuse, sait encore donner au siècle des Lumières des résultats de tout intérêt.

\section{AUTEUR}

\section{NADIA MINERVA}

Université de Perugia, Italie 